\title{
Using desktop virtual environments to investigate the role of landmarks
}

\author{
Petra Jansen-Osmann* \\ Heinrich-Heine-University Düsseldorf, Institute of Experimental Psychology, Universitätsstrasse 1, \\ 40225 Düsseldorf, Germany
}

\begin{abstract}
Recent research on spatial cognition has used computer-simulated three dimensional environments to create appropriate laboratory settings when trying to examine processes of spatial orientation. One way to evaluate "virtual environments" is to replicate results of experiments which were originally conducted in traditional laboratory or in real world settings. The experiment which is reported here investigates the role of landmarks when acquiring route knowledge in a system of paths. The design follows an earlier experiment by Cohen and Schuepfer [Child development 31 (1980) 1065]. It can be shown, that landmarks play an important role in the system of paths: landmarks which are combined with turns towards the destination are more likely to become strategic nodes in the network than those which are not connected. Beside these results, a software designed to build appropriate environments is shown and its usability is demonstrated. The use of virtual environments provides both economical and flexible design of realistic experimental settings, as well as a valide recording of behavior. (C) 2002 Published by Elsevier Science Ltd.
\end{abstract}

Keywords: Spatial cognition; Landmark; Desktop virtual environment

Humans acquire spatial knowledge in a more primary manner by traveling through environments and in a more secondary way by the use of maps, photographs, verbal descriptions and, more recently, virtual environments. Though virtual environments are used in many areas, including the fields of engineering, architecture, design, entertainment and education, the use of these environments plays a dominant role in the investigation of spatial processes. This technology allows the simulation of three-dimensional environments on a computer: humans can experience those environments by active exploration, VR conveys a strong impression of movement through space. These virtual spaces can be used to simulate any setting,

* Tel.: +49-211-811-2270; fax: +49-211-811-5037.

E-mail address: petra.jansen-osmann@uni-duesseldorf.de (P. Jansen-Osmann). 
and allow target-driven interaction by the subjects. First of all, there are two types of VR-systems: desktop and immersive display systems. The latter uses devices like head mounted displays (HMDs) and tracking systems, which give the user the impression of being completely "immersed" in the virtual world, without any trace left of the real world. In contrast to this, desktop systems display the virtual world on a monitor. There are two intermediate solutions between desktop and immersive display systems: first, the use of a projection-screen, and second three-dimensional monitors. The latter, which is also called a mixed-reality application, allows a threedimensional impression of the world to be explored. Virtual and real three-dimensional objects can be observed simultaneously and interact (Bertuch, 2001).

Desktop as well as immersive virtual environments seemed to be a practical medium for the simulation of spatial environments: Spatial relations can be varied quickly and in an economic manner, the participants can operate self-determined, and both real and fictional environments can be simulated (cf. Goldin \& Thorndyke, 1982). Within the spatial cognition research, virtual environments are used to investigate the navigation behavior in virtual environments (Darken \& Silbert, 1996; Ruddle, Payne, \& Jones, 1999) and the processes which underlie the solution of spatial tasks, e.g. path-integration, under the exclusion of proprioceptive information (May \& Klatzky, 2000). It has been shown that the acquisition of route knowledge and survey knowledge in virtual environments is possible (Bliss, Tidwell, \& Guest, 1997; Gillner \& Mallot, 1998). Furthermore, subjects can estimate directions (Albert, Rensink, \& Beusmanns, 1999) and distances (Jansen-Osmann \& Berendt, in press; May, Péruch, \& Wartenberg, 1997). The significance of virtual environments in spatial cognition research which exists in the economic and realistic designing gets more and more appreciated (Péruch, Gaunet, Thinus-Blanc, \& Loomis, 2000).

The goal of this paper is to evaluate the use of virtual desktop environments in spatial cognition research. One form to do this is to replicate experiments which were formerly done in laboratory experiments or in real world settings.

In this study we replicated in a desktop virtual environment an experiment by Cohen and Schuepfer (1980) by using Superscape VRT software. Presently there are various software products available which allow the creation of virtual environments but which differ in regard to their variability concerning spatial parameters and the degree to which they allow the behavior of persons in virtual space to be traced. Without doubt, the number of VR products will increase in the near future. The choice of software depends on the available hardware and the kind of environment to be simulated. In this study, Superscape VRT software was selected, because it is particularly suitable for a PC, even for a mid-range system. The software includes specific editors which allow variation of systems of paths, the systematic localization of static objects, e.g. houses and trees, or moving objects, e.g. cars or pedestrians which can be designed flexibly in their structure and texture. Importing soundfiles and placing them in the system of paths is also possible. The user can actively navigate through the virtual environment using a joystick, and the path they choose can be recorded. The program also allows passive navigation through space or showing subjects the system of paths from a survey presentation. The software 
offers a complete graphic user interface for the creation of virtual environments. With the help of the various editors the designer can create virtual worlds in realtime and manipulate them systematically.

An example of the possible uses of this program for designing a particular virtual world and systematically tracing learning in virtual learning environments is the research in the present study on the role of landmarks in spatial cognition. Mentioned above, the study described here follows a research methodology used by Cohen and Schuepfer (1980) to investigate orientation behavior of young people in space. The authors studied the question of how route knowledge is acquired and what the role of the localization of objects (landmarks) in the system of paths is. Three experimental groups grouped by age (average age 7.8, 11.9, and 23.6 years) with 20 participants each were given the task of learning the route through a system of corridors, in which there were landmarks with different functions (adjacent to the correct, the incorrect, or to no turn) in different sections as possible navigation help (cf. Fig. 1). Cohen and Schuepfer (1980) brought about systematic variation by way of discontinous presentation of scenes, which is of course beset by the artificiality and complexity typical of such laboratory experiments. In each scene that was projected as a slide on a projection-screen, participants saw consecutive sections of the complete way with various bifurcations and landmarks of different directional importance. The landmarks consisted of various objects. After a slide had been presented, participants were asked to decide which way they thought should be taken to reach the destination. If they had chosen the right way, they were shown the next slide. If not, they were asked to try again. This sequence decision task was complete when a participant managed to predict the way correctly six consecutive times. In the following test phase, pictures of the maze were shown without objects and participants were asked to indicate the correct turn in each case and, furthermore, to state which objects had been at the particular turn. Beside the developmental find-

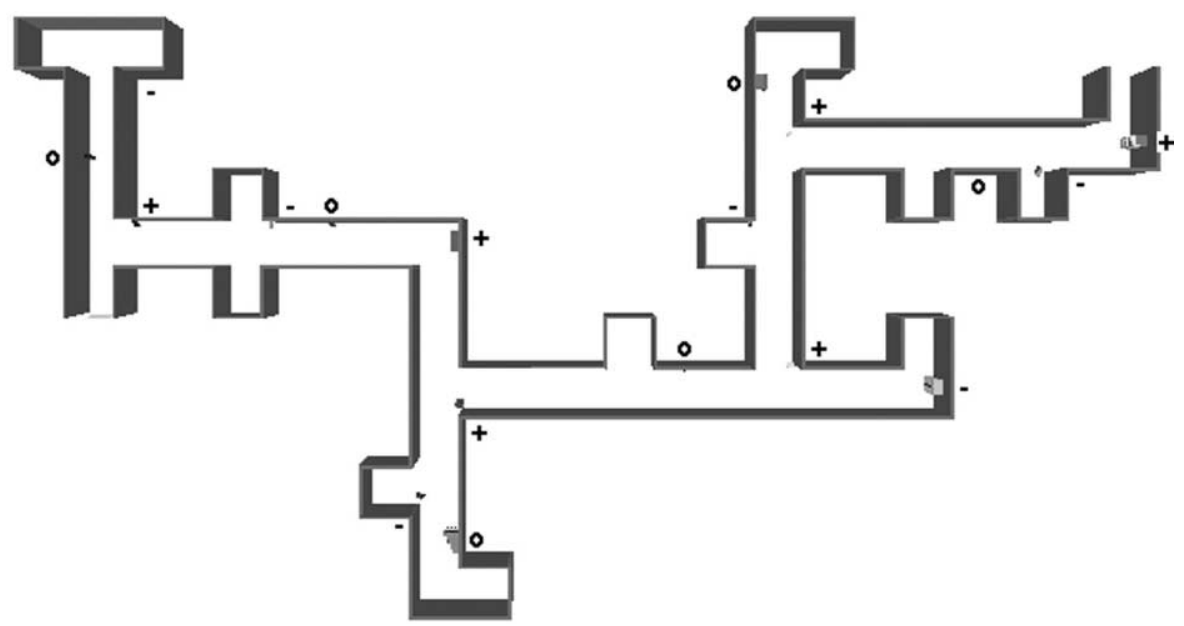

Fig. 1. Bird's eye view of the maze ("+ " denotes a landmark adjacent to a correct turn, "-" denotes a landmark adjacent to a wrong turn, "o" denotes a landmark adjacent to no turn). 
ing that the youngest participants had the greatest difficulty when acquiring spatial knowledge, it could be shown in the recall test for all age groups that landmarks that indicated a correct turn were significantly better localized than those that were irrelevant for finding a direction or were located at a wrong turn.

In the present study it was investigated first how well adults could find their way through the maze by actively navigating through it in a desktop virtual environment. In contrast to the study of Cohen and Schuepfer (1980) half of the participants had to explore the maze with landmarks, the other half without landmarks until reaching a learning criterion. Furthermore, we examined whether the findings of the recall test could be replicated.

\section{Method}

\subsection{Participants}

Participants were 10 students (average age: 25.85 years) at the Gerhard-MercatorUniversity in Duisburg, Germany.

\subsection{Materials}

The experiment was conducted on a PC with Superscape's VRT 4.00 software. A maze was programmed that corresponded to the system of paths Cohen and Schuepfer (1980) used in their study. The maze was presented to participants on a 17 inch screen. They "walked" through the maze by using a joystick. Fig. 1 presents a hardcopy of a bird's eye view of the maze.

The maze consisted of six main corridors. Two secondary corridors branched off from each main corridor and ended in a cul-de-sac. The maze itself was identical in all experimental conditions except for its interior appearance. One condition did not contain any landmarks whereas the other condition contained 18 landmarks of various kinds and shapes (e.g. a ball, screen, picture, pen etc.) The landmarks were located just like in Cohen and Schuepfer's (1980) system of paths at the points marked in Fig. 1. In Fig. 1, a "+" denotes a landmark adjacent to a correct turn, a "-" denotes a landmark adjacent to a wrong turn and an "o" denotes a landmark adjacent to no turn. The paths taken by the participants were automatically recorded and made visible and available for analysis as a trace of arrows in a bird's eye view of the maze.

\subsection{Procedure}

The experiment was conducted in individual 15-30 min sessions. Before the experiment began, participants were given the opportunity to familiarize themselves with the joystick. As soon as they were sufficiently familiar with the joystick, the experiment itself began. In the first part of the experiments participants were asked to travel through the maze until they had reached the destination in two consecutive 
attempts without errors. The number of learning trials necessary until the criterion was reached was recorded as well as the number of errors in each trial. An error was defined as choosing a wrong turn. Fig. 2 shows the opening shot of the maze with landmarks.

The menu bar shows the possible alternatives. Upon mouseclick of one of the four large buttons one can start the experiment, playback the path taken, analyze the trial from the bird's eye view, or delete the analysis. The buttons "world 1" and "world 2" allow switching between the two mazes (the one with landmarks and the one without landmarks).

In the final test phase, the participants who had traveled the maze with landmarks were asked to travel the one without landmarks and were asked to recall the places and sorts of landmarks they could remember.

\section{Results}

The path participants took was recorded for analysis. The arrow trace from the bird's eye view of the maze shows whenever they strayed from the way to the destination (Fig. 3)

Table 1 shows the number of learning trials until the criterion was reached and the number of wrong turns per trial (errors) for the different conditions. Error-free trials were not used in computing these performance parameters.

The statistic shows that the maze is traveled more often when it did not contain landmarks than when the participant can use landmarks to navigate $\left(t_{(8)}=2.19\right.$,

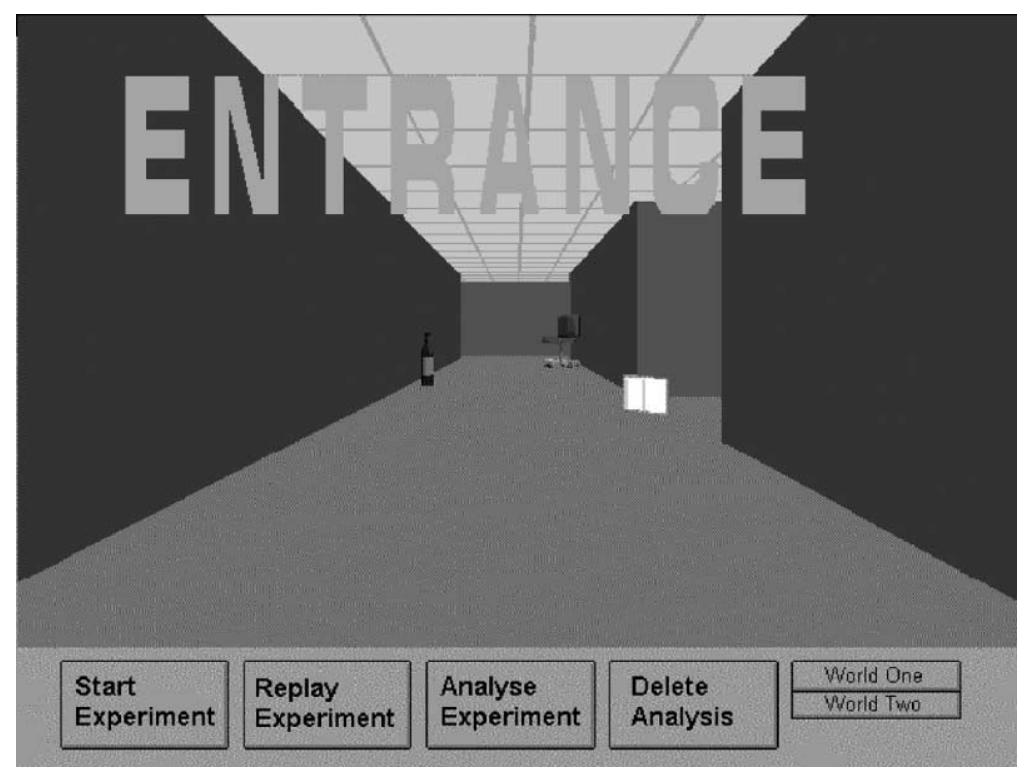

Fig. 2. Opening shot of the maze. 
Table 1

Number of learning trials until the criterion was reached and number of wrong turns per trial (mean, standard deviation, maximum, and minimum for the different kind of mazes)

\begin{tabular}{llcccc}
\hline Performance index & Kind of maze & Mean & $\begin{array}{l}\text { Standard } \\
\text { deviation }\end{array}$ & Max. & Min. \\
\hline Number of learning trials & Without landmarks & 4.6 & 2.15 & 8 & 2 \\
& With landmarks & 2.4 & 0.6 & 3 & 2 \\
Number of wrong turns & Without landmarks & 15.8 & 5.49 & 22 & 7 \\
& With landmarks & 11.4 & 3.61 & 18 & 5 \\
\hline
\end{tabular}

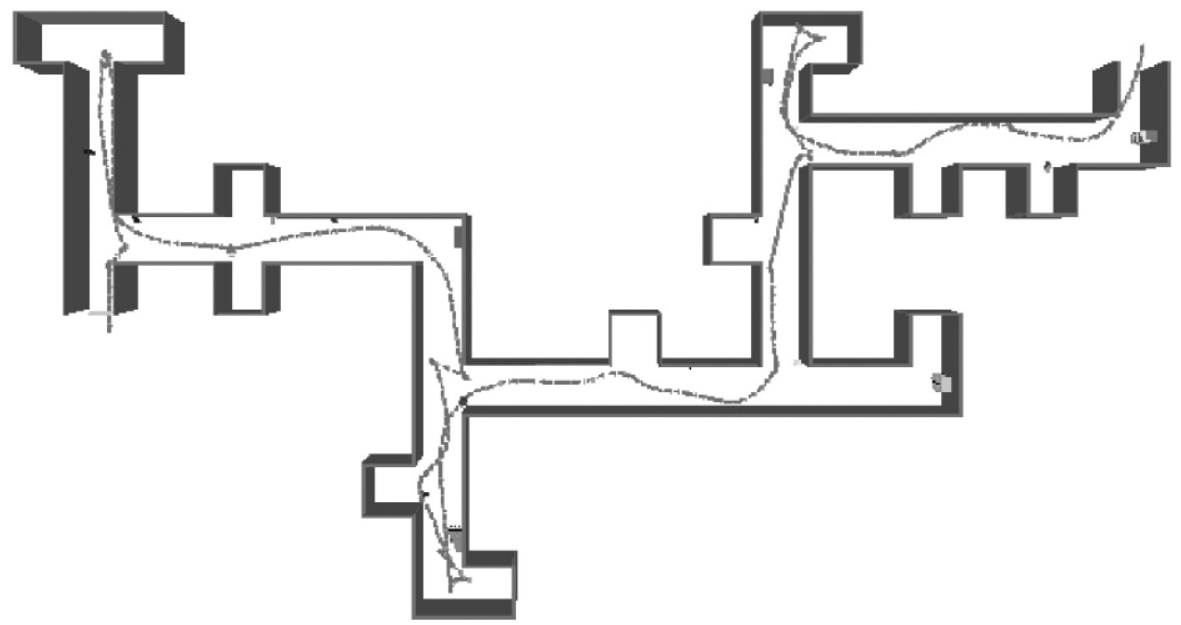

Fig. 3. Tracking of a participant's way through the maze (here: maze with landmarks).

$P<0.05)$. The number of wrong turns per trial is also lower when participants could make use of landmarks. However, this difference is not significant.

The learning curves that describe the process of acquiring spatial knowledge are shown in Fig. 4.

Fig. 4 indicates that the average number of wrong turns per trial declines steeply under both conditions between the first and the second trial. This trend continues into the third trial when subjects are given the benefit of landmarks. Without landmarks, the number of errors only declines gradually after the second trial.

The number of landmarks correctly reproduced after meeting the criterion on the path with landmarks is shown in Table 2.

Table 2 shows that pro-directional landmarks (adjacent to the right turn) can be significantly better reproduced than counter-directional (adjacent to the wrong turn) ones $\left(t_{(4)}=4.15, P<0.05\right)$ or landmarks without directional relevance $\left(t_{(4)}=3.88\right.$, $P<0.05)$. This result resembles the result obtained in the study of Cohen and Schuepfer (1980): In their study college students remembered on average 5.4 


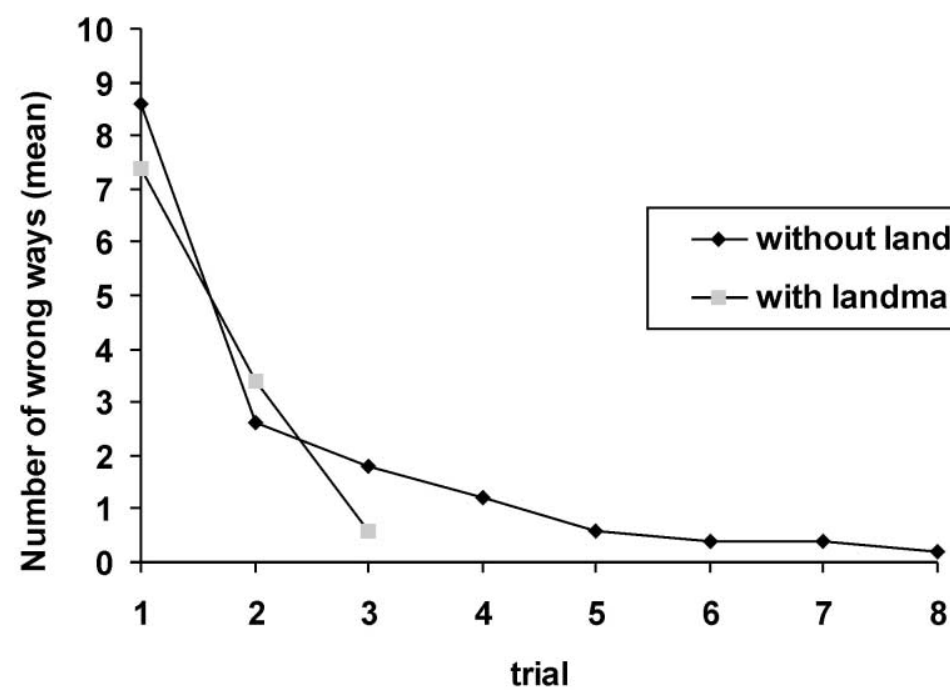

Fig. 4. Learning curves for the navigation through the maze (mean number of wrong turns per trial).

Table 2

Number of correctly reproduced landmarks after meeting the criterion (mean, standard deviation, maximum, and minimum for the different kind of landmarks)

\begin{tabular}{lllll}
\hline Kind of landmark & Mean & Standard deviation & Max. & Min. \\
\hline Adjacent to a right turn & 4.6 & 1.49 & 6 & 2 \\
Adjacent to a wrong turn & 1.2 & 0.98 & 3 & \\
Adjacent to no turn & 1 & 0.44 & 2 & 0 \\
\hline
\end{tabular}

landmarks adjacent to the right turn (4.6 in the study presented here), 1.35 landmarks adjacent to a wrong turn (1.2 in this study) and 0.8 landmarks adjacent to no turn (1.0 here).

\section{Discussion}

Cohen and Schuepfer's (1980) findings concerning the relevance of landmarks in learning a route, could be replicated even with a small sample size in a virtual environment on a mid-range computer system. Whereas Cohen and Schuepfer (1980) obtained their results with a design that appears extraordinarily artificial and complex, the participants in this study could actively navigate. Landmarks do indeed aid orientation when finding the way, and a route with landmarks is learned faster than one without landmarks. The role landmarks play in the system of paths is relevant in this context: landmarks which are connected with a turn towards the destination are more likely to be strategic nodes in the network than other landmarks (cf. Lynch, 1960). They are also remembered better than other landmarks. The cognitive map 
participants acquired after navigating the maze that contained landmarks additionally facilitates access to pro-directional landmarks since these have the function as nodes in the organizational structure of the cognitive map.

That the results of Cohen and Schuepfer's (1980) experiment could be replicated in a desktop virtual environment seem to confirm that the same cognitive processes operate in real and virtual environments. This is in accordance with the results from an experiment of Ruddle, Payne, and Jones (1997), who replicated in virtual environments most of the findings obtained by Thorndyke and Hayes-Roth (1982) in real settings: participants were found to be equally good at performing direction and relative distance estimates. Furthermore, in our own work we could replicate a result which were obtained by Sadalla and Magel (1980): a route with a higher number (7) of turns was estimated longer than a route of the same length with less (2) turns (Jansen-Osmann \& Berendt, in press).

Unlike in Cohen and Schuepfer's (1980) experiment in which spatial information was presented in discrete steps as successive presentation of different sections of the way, participants traveled the experimental space continuously in our setup. Learning a way in this manner is much more closely related to acquiring route knowledge in the real world. There is still a certain amount of artificiality associated with the desktop virtual reality used here because the virtual space is not really traveled but only experienced in its sections by use of a joystick. However desktop systems like they are used in the study presented here are only the first step to complete immersive display systems. The use of faster workstations and head-mounted displays with corresponding tracking systems will give an even stronger impression of reality, also relative to subjects' own locomotion.

As far as the respective advantages and disadvantages of laboratory and field experiments are concerned, the use of VR allows for the manipulation of field settings and therefore can be used to approach the naturalness of field experiments while maintaining a high degree of experimental control. The old dichotomy of artificial vs. natural to distinguish between laboratory and field experiments will no longer be relevant once VR has been developed further. Experimental parameters can be widely and systematically varied in VR experiments. Software variations like the structure of the system of paths, design of routes, kinds and localization of static and mobile objects can be generated with little expense of time, effort and money. Furthermore, the Virtual Reality technique provides continuous measurements during navigation. The use of virtual environments in research avoids constraining limits of real-world experimental situations (Péruch, Gaunet, Thinus-Blanc, \& Loomis, 2000).

The virtual reality technology introduced here allows

- fast variation of visual details and local spatial relationships,

- users the chance to interact with the simulated environment by letting them choose paths, perspectives and speed of travel,

- bird's eye view or inside view of a virtual world,

- fast and economical simulation of the desired spatial environment, and

- simulation of real as well as fictional environments. 
These are the basic constraints on a simulation medium (cf. Goldin \& Thorndyke, 1982).

In summary, these results illustrate that the cognitive components of the processing of spatial information could be investigated in desktop virtual environments. Knowing that, we can go further varying these environments for the investigation of spatial processes. These studies are just beginning but seem to be fruitful. More and more complex and realistic virtual environments will contribute to simplify the investigation of spatial knowledge.

\section{Acknowledgements}

I would like to thank Frank P. Schulte for his help writing this manuscript.

\section{References}

Albert, W. S., Rensink, R. A., \& Beusmanns, J. (1999). Learning relative directions between landmarks in a desktop virtual environment. Spatial cognition and computation, 1, 131-144.

Bertuch, M. (2001). 3D-Displays werden perfekter und aufwendiger. C't, 19, 33.

Bliss, J., Tidwell, P., \& Guest, M. (1997). The effectiveness of virtual reality for administering spatial navigation training to firefighters. Presence, 6, 73-86.

Cohen, R., \& Schuepfer, T. (1980). The representation of landmarks and routes. Child Development, 31, 1065-1071.

Darken, R. P., \& Silbert, J. L. (1996). Navigating large virtual spaces. International Journal of HumanComputer Interaction, $8,49-71$.

Gillner, S., \& Mallot, H. (1998). Navigation and acquisition of spatial knowledge in a virtual maze. Journal of Cognitive Neuroscience, 10, 445-463.

Goldin, S. E., \& Thorndyke, P. W. (1982). Simulating navigation for spatial knowledge acquisition. Human Factors, 24, 457-471.

Jansen-Osmann, P., \& Berendt, B. Investigating distance knowledge using virtual environments. Environment and Behavior, in press.

Lynch, K. (1960). The image of the city. Cambridge, MA: MIT Press.

May, M., \& Klatzky, R. (2000). Path integration while ignoring irrelevant movement. Journal of Experimental Psychology: Learning, Memory, and Cognition, 26, 169-186.

May, M., Péruch, P., \& Wartenberg, F. (1997). Raumorientierung in virtuellen Umgebungen. In R. Kluwe (Ed.), Strukturen und Prozesse intelligenter Systeme. Wiesbaden: Deutscher Universitäts-Verlag.

Péruch, P., Gaunet, F., Thinus-Blanc, C., \& Loomis, J. (2000). Understanding and Learning virtual spaces. In R. M. Kitchin, \& S. Freundschuh (Eds.), Cognitive mapping: past, present and future. London: Routledge.

Ruddle, R. A., Payne, St.J., \& Jones, D. M. (1997). Navigationg buildings in desk-top virtual environments: experimental investigations using extended navigational experience. Journal of Experimental Psychology: Applied, 3, 143-159.

Ruddle, R. A., Payne, St.J., \& Jones, D. M. (1999). The effect of maps on navigation and search strategies in very-large-scale virtual environments. Journal of Experimental Psychology: Applied, 5, 54-75.

Sadalla, E. K., \& Magel, S. G. (1980). The perception of traversed distance. Environment and Behavior, 12, $65-79$.

Thorndyke, P. W., \& Hayes-Roth, B. (1982). Differences in spatial knowledge acquisition from maps and navigation. Cognitive Psychology, 14, 560-589. 
Vitae

Petra Jansen-Osmann holds a Master's Degree in Biological and Social Anthropology and Psychology from Johannes-Gutenberg-University Mainz and a PhD in Cognitive Psychology from Gerhard-Mercator-University Duisburg. She is currently a research fellow at the Institute of Experimental Psychology at HeinrichHeine-University, Düsseldorf, Germany. Her research is concerned with human spatial cognition and behavior in natural and virtual environments and motor development. 